\title{
Sexualidad en Adultos Mayores: Estereotipos en el Alumnado Universitario del Grado de Educación Primaria
}

\author{
Encarnación Pedrero-García ${ }^{1}$, Pilar Moreno-Crespo ${ }^{2}$ y Olga Moreno-Fernández ${ }^{3^{*}}$ \\ (1) Facultad de Ciencias Sociales. Universidad Pablo de Olavide. Carretera Utrera, Km. 1, Sevilla, \\ España. (e-mail: epedgar@upo.es) \\ (2) Facultad de Ciencias de la Educación. Universidad de Huelva. Avenida 3 de marzo s/n, Huelva, \\ España. (e-mail: pilar.moreno@dedu.uhu.es) \\ (3) Facultad de Ciencias de la Educación. Universidad de Sevilla. C/ Pirotecnia s/n, Sevilla, España. \\ (e-mail: omoreno@us.es) \\ *Autor a quien debe ser dirigida la correspondencia
}

Recibido Jul. 10, 2017; Aceptado Sep. 25, 2017; Versión final Nov. 3, 2017, Publicado Abr. 2018

\begin{abstract}
Resumen
El objetivo de la investigación es identificar si el alumnado de magisterio posee estereotipos sobre la sexualidad de las personas adultas mayores. Para ello se realizó un estudio descriptivo transversal, utilizando el Cuestionario de Actitudes hacia la sexualidad en la vejez (CASV). Aunque los resultados muestran que el alumnado presenta bajos niveles de estereotipos en siete de los trece ítems, se encontró cuatro ítems que presentan un grado medio, así como dos ítems donde se representa un grado alto y muy alto. Algunos resultados a las preguntas son: P7: Todas las mujeres mayores son frígidas; $98,2 \%$ vs. $1,8 \%$; P6: Los hombres mayores son impotentes; $80,0 \%$ vs. $20,0 \%$; y P1: Tienen menos interés por las relaciones sexuales; $48,6 \%$ vs. $51,3 \%$. Se concluye que siguen existiendo estereotipos entre los encuestados, aunque con diferentes grados.
\end{abstract}

Palabras clave: educación de personas adultas mayores; sexualidad; estereotipos; formación universitaria; formación inicial

\section{Sexuality in Older Adults: Stereotypes in University Students of the Primary Education Degree}

\begin{abstract}
The objective of the research was to identify whether students of the area of education (future scholl teachers) have stereotypes about the sexuality of older adults. For this, a cross-sectional descriptive study was carried out, using the Attitudes Questionnaire towards sexuality in old age (CASV). Although the results show that students have low levels of stereotypes in seven of the thirteen items (P7: All the older women are frigid, $98.2 \%$ vs. $1.8 \%$ ), we find four items that present an average grade (P6: Older men are impotent, $80.0 \%$ vs. $20.0 \%$ ), as well as two items where high and very high grade is represented (P1: [...] they are less interested in sexual relations, $48.6 \%$ vs. $51.3 \%$ ). There are still stereotypes among respondents, albeit with varying degrees.
\end{abstract}

Keywords: education of older adults; sexuality; stereotypes; university education; initial training 


\section{INTRODUCCIÓN}

¿Por qué investigar los estereotipos en el grupo poblacional de los adultos mayores? El fenómeno del envejecimiento poblacional se sucede en buena parte del planeta, principalmente en los países desarrollados, con un patrón que es seguido por los países en vías de desarrollo. Ya es una realidad que el peso poblacional, demográficamente hablando, lo sustentan las personas con más de 65 años. Nos encontramos, por lo tanto, ante un planeta en un proceso constante e implacable de envejecimiento de la población. El reto ante el que se encuentra la actual sociedad "encanecida" es promocionar un entorno que favorezca el desarrollo en las mejores condiciones de la sociedad en general y de las personas adultas mayores en particular, así como tratar de superar los estereotipos predominantes en la actualidad (Amador, Moreno-Crespo y Cárdenas, 2006; Moreno-Crespo, 2010, 2011, 2015; Moreno-Crespo y Pérez-Pérez, 2014).

Con frecuencia, los conceptos de estereotipos y prejuicios en Psicología Social, se suelen relacionar de manera casi automática o incluso confundirse. Sin embargo, aunque es obvio que están fuertemente relacionados la literatura enfatiza que se trata de conceptos claramente distintos. Por ello, consideramos que ambos constructos forman parte de las actitudes, de manera que los estereotipos hacen referencia al componente cognitivo de las mismas, mientras que el prejuicio es el componente afectivo y ambos, se relacionan a su vez, con el componente comportamental que se materializa en las conductas discriminatorias (Eagly y Chaiken, 1998; Fishbein y Ajzen, 1974 citados en De Lemus, 2007). De acuerdo con esta idea, seguimos la definición de estereotipo propuesta por Morales y Moya (1996: 163) como "un conjunto de creencias, compartidas acerca de los atributos personales que poseen los miembros de un grupo". Además, consideramos que estas creencias se conforman en el pasado y se transmiten a través del proceso educativo, sin apenas transformación, con el paso del tiempo (Pedrero, 2002). Este proceso de transmisión afianza una de las características más significativas de los estereotipos: su resistencia al cambio y el efecto anticipador de la conducta. De esta forma, los estereotipos o prejuicios se convierten en esquemas funcionales que activan o dirigen la actuación mucho más que la propia realidad. Por ello, la importancia que tiene todo estereotipo es su transmisión a través de la educación y su perdurabilidad en el tiempo, preocupándonos principalmente los estereotipos vinculados al envejecimiento.

En nuestra sociedad actual, se aprecia una clara concepción negativa de la vejez. En un estudio realizado con 1200 personas de más de 18 años, los adjetivos asignados a los adultos mayores fueron: pasivos, lentos, tristes o serenos (Fernández-Ballesteros, 2000). Triadó y Villar (2006) por otra parte afirman que las características que se suelen atribuir a las personas mayores son: están enfermas y dependen de otras personas, tienen algún tipo de deterioro cognitivo, son incapaces de aprender cosas nuevas, se vuelven rígidas y difíciles de tratar, son conservadoras y cerradas al cambio, están socialmente aisladas y lo que desean es descansar... Estos estereotipos, enraizados en nuestro contexto cultural respecto a los mayores funcionan como esquemas mentales previos, pudiendo provocar en su caso actitudes "antimayores" o "viejistas", que por falta de objetividad son inadecuadas a la correcta valoración de este sector de la población (García et al., 2002). Además, son compartidos por niños, adolescentes, jóvenes, adultos e incluso muchas personas mayores, lo que favorece que pueda llegar a ser así porque es lo que se espera de las personas mayores (López-Sánchez, 2012).

Hasta tal punto pueden llegar estas actitudes de rechazo que Butler (1969) citado en Bárcena, Iglesias, Galán y Abella (2009: 47) definió el término "edadismo" para referirse a los estereotipos sistemáticos y discriminatorios contra las personas por el simple hecho de ser mayores y que se reflejan en conductas tales como el desdén, el desagrado, el insulto o, simplemente, evitando la cercanía y el contacto físico. El edadismo, por lo tanto, hace referencia al mantenimiento de estereotipos o actitudes prejuiciosas hacia una persona, únicamente por el hecho de ser mayor. Pedrero (2002) realiza una descripción de aquellas características estereotipadas capaces de definir a las personas mayores en nuestro contexto actual, son personas atrapadas a sus costumbres a las que no les suele gustar los cambios ni las novedades, piensan con frecuencia en su pasado, son pausadas en sus acciones y pensamientos, pueden mostrarse recluidas en sí mismas y en su entorno, son difíciles de tratar por la irritabilidad con la que se muestran, suelen estar aquejadas de enfermedades, en muchos casos causa de la inactividad, lo que les impide disfrutar de determinados placeres, su capacidad e interés por las relaciones sexuales decae o incluso desaparece y su capacidad de producción disminuye. Estos rasgos con los que se definen a los adultos mayores no dejan de ser el reflejo de un substrato cultural que determina esta forma de pensamiento en nuestro entorno.

Está muy extendida la creencia de que los cambios que se producen en la vejez son exclusivamente negativos, sostenidos por la pérdida de habilidades y capacidades. Incluso, algunas personas de más de sesenta y cinco años se niegan a reconocer el periodo en el que se encuentran, considerándose de mediana edad. Esta situación provoca que lo viejo no se valora positivamente, incluso es infravalorado (Allan, Johnson y Emersona, 2014; Aguirre et al., 2001; Escarbajal y Martínez, 2012; García et al., 2002; García y Benito, 2003; López-Sánchez y Olozabal, 2005; Lucas-Matheu, 2013; Moragas y Linz, 1991), aunque consideramos que debería ser al revés, un valor añadido, dada la acumulación cognitiva que conlleva y el uso social que de ella se derivaría. 
Todo lo dicho vendría a potenciar la idea de la vejez como un problema de carácter social, idea que está bastante extendida entre la población. Frente a ello cabría la opción de reconocer a este colectivo como grupo a respetar con un importante rol social que desempeñar. A su vez, el lenguaje, nos ayuda a potenciar o disminuir la discriminación (Bergman y Bodner, 2015). En el caso de la palabra "anciano" nos encontramos con que conlleva unas connotaciones socialmente negativas, unas falsas concepciones que conviene conocer. En diferentes ambientes, incluidas aquellas profesiones dedicadas a la atención en el ámbito de las personas mayores, nos podemos encontrar con estas ideas negativas sobre las características que podrían definir a las personas mayores (Escarbajal et al., 2015). Estas falsas concepciones pueden igualar vejez a decadencia y deterioro de todo tipo: físico, mental, funcional, etc., hecho que no coincide con la realidad. La sociedad desde tiempo remoto ha creado unos formatos mentales perpetuando una imagen equivoca de las categorías que vendrían a definir esta etapa de la vida. Nuestros estereotipos negativos no se ajustan a la realidad de la mayoría de los adultos mayores, de esta forma, ni ellos mismos se reconocen en la definición de anciano dando el valor social negativo que se le atribuye (Pedrero, 2002).

Llegados a este punto se plantea cuál es la importancia de la existencia de estereotipos vinculados a las personas adultas mayores. Y se encuentra que hay numerosas investigaciones realizadas sobre la influencia de los estereotipos negativos en la vejez, entre las que podríamos destacar la realizada por Levy y Myers (2004), en la que concluyen que estas representaciones sociales influyen tanto en su capacidad cognitiva como en su memoria, pudiendo causar además estrés y pronosticar una disminución en la esperanza de vida. Por otra parte, también señalaron que aquellos mayores que tenían una opinión positiva sobre su propio envejecimiento disfrutaron de una salud funcional mejor que los que tenían una opinión más negativa. Por ello es tan importante combatir estos estereotipos ya que afectan no solamente a nivel social sino a nivel individual a las personas que se encuentran en esta etapa del ciclo vital.

¿Qué sucede si seleccionamos la sexualidad como centro de interés en relación a los estereotipos hacia las personas mayores? Que la situación se recrudece cuando se relaciona sexualidad y vejez, ya que el mito principal es considerar erróneamente a este ciclo de la vida como una etapa prácticamente asexuada (LucasMatheu, 2013). Algunos de los mitos más comunes respecto a la sexualidad en la persona mayor, se refieren a aspectos como una menor actividad sexual a medida que avanza la edad, la falta de deseo y atractivo, la asociación del sexo con la reproducción, así como la negación del deseo sexual a la mujer mayor (Cerquera et al., 2012). Si queremos ser más exhaustivos podríamos recoger los principales mitos como hizo Pedrero (2003: 192-193) en torno a la sexualidad del adulto mayor y nos encontraríamos con afirmaciones referidas a los varones tales como que ya no tienen erecciones, son incapaces de eyacular, no pueden alcanzar el orgasmo, son asexuados... En cuanto a las mujeres estos mitos se encuentran muy relacionados con la menopausia, de modo que se afirman cuestiones como que la mujer menopaúsica ya "no es mujer" o "ya no sirve", ya no tiene deseo sexual en la menopausia, pierde atractivo sexual para el varón, pierde sensibilidad en sus genitales, etc., en definitiva, parece que al entrar en esta nueva etapa ya no hay actividad sexual tal y como afirman estos mitos. Freixas y Luque (2009) explican la presencia de estos mitos en base a las diferencias de género sexuales y para ello hablan del doble rasero sociocultural por el cual se ofrece permisividad a los varones para actuar como agentes sexuales, pero desvaloriza y estigmatiza a las mujeres que responden a sus necesidad y deseos sexuales, colmándolas de términos denigrantes que no se utilizan con los varones en las mismas circunstancias.

En cuanto a las investigaciones realizadas en las últimas décadas, el catedrático de sexología Félix LópezSánchez (2012) afirma que han avanzado bastante, pero aún siguen mostrando numerosas limitaciones referidas tanto a nivel conceptual como metodológico. Por ello, mientras no tengamos estudios longitudinales que sigan el curso de la vida sexual de las personas y nos permitan conocer lo que puede haber de universal en los cambios evolutivos propiamente dichos y diferenciarlo de lo que puede ser explicado por cambios generacionales, no tendremos una visión más objetiva y menos estereotipada de la conducta sexual en la vejez. Por tanto, podemos trabajar en establecer una nueva cultura del envejecimiento, una cultura que considere a las personas de edad agentes y beneficiarios de su progreso, que esté basada en un modelo de desarrollo, participación y autorrealización del ser humano en esta etapa de la vida. La calidad de vida que se obtenga en la adultez dependerá de su experiencia vital previa, es decir, de las posibilidades que se hayan tenido de llevar un estilo de vida sano, de la educación permanente, el desarrollo de aptitudes, de los incentivos para ahorrar, de los planes de pensiones y de las medidas para fomentar la creación de empresas y redes familiares y comunitarias que incluyan a personas de todas las edades (García et al., 2002).

En la revisión de la literatura sobre estereotipos sobre la sexualidad en personas adulas mayores, encontramos pocas investigaciones que estudian la percepción del alumnado universitario de titulaciones relacionadas con el campo de la educación. En concreto, se ha seleccionado el Grado de Maestro en Educación Primaria, ya que profesionalmente el maestro es un agente socializador y transmisor de cultura, junto con que, en España, es el perfil académico necesario para impartir educación de personas adultas 
reglada (Matas et al., 2016). Es decir, se ha seleccionado una profesión que entre sus salidas profesionales contempla la posibilidad de ser educador o educadora de personas adultas. En concreto, nos hemos decantado por la Universidad de Sevilla por tener más de cinco siglos de historia, lo que la sitúa entre las diez universidades públicas más antiguas de España que continúan en activo. Por lo tanto, nos interesa identificar si el alumnado de magisterio posee estereotipos sobre la sexualidad de las personas adultas mayores ya que la detección de estos estereotipos negativos sobre este grupo poblacional facilitará desarrollar programas formativos específicos que garanticen un profesional objetivo con la realidad de esta población (Díaz et al., 2009; Van Dussen y Weaver, 2009; Matas et al., 2017).

\section{METODOLOGÍA}

A partir de la conceptualización analizada surge un interrogante esencial al que este trabajo pretende dar respuesta: ¿Posee el estudiantado de magisterio estereotipos sobre la sexualidad de las personas adultas mayores? El presente trabajo busca por tanto identificar si el estudiantado de magisterio de la Universidad de Sevilla posee estereotipos sobre la sexualidad de las personas adultas mayores. La fuente de datos primarios se obtuvo de un cuestionario de escala tipo Likert.

Para el estudio de campo se recurrió a la Universidad de Sevilla, por ser una de las más antiguas de España. La muestra estuvo conformada por 110 alumnos del Grado de Magisterio. Educación Primaria ( $\mathrm{N}=110)$, de entre 19 y 37 años de edad, situándose el perfil de edad mayoritariamente entre los 20 y los 22 años. En cuanto al sexo, el $71,8 \%$ de la muestra encuestada son mujeres y un $28,2 \%$ hombres. Se realizó un estudio cuantitativo, a partir de un muestreo exploratorio, no probabilístico por conveniencia (Canales, 2006; Hernández, Fernández y Baptista, 2006) orientándose a la selección de aquellas unidades muestrales que garantizaran cantidad y calidad de información relevante.

\section{Instrumento}

El instrumento utilizado en la presente investigación se basa en el Cuestionario de actitudes hacia la sexualidad en la vejez (CASV) (Orozco y Rodríguez, 2006). Melguizo-Herrera et al. (2015) indican que la escala CASV permite la cuantificación válida y confiable de las actitudes ante la sexualidad en los adultos mayores. Esta escala contiene 14 preguntas cerradas. De las 14 preguntas del CASV se seleccionaron 11, y se añadieron 2 cuestiones más de interés para la investigación. Del CASV se eliminaron los siguientes ítems: La andropausia marca el inicio de la vejez, la menopausia marca el final de la vida sexual de la mujer, y las enfermedades más frecuentes en la vejez limitan la actividad sexual. Los motivos por los que se decidió eliminar estos ítems fue porque el que las preguntas estén orientadas a adultos mayores implica que se trata de personas que se encuentran a nivel biológico en estos estadios, es decir, en la menopausia o en la andropausia (el debate sobre la existencia (Leiva, Arguedas, Hidalgo y Navarro, 2013; Sapetti, 2013) o no existencia (Vermulen y Kaufman, 1995) de la andropausia a nivel científico se encuentra aún abierto). En cuanto a las enfermedades más recientes en la vejez consideramos que podía dar lugar a confusión el hecho de que no se especificara implícitamente a que enfermedades se estaba refiriendo ¿Cuáles son las enfermedades más comunes en los adultos mayores? Por su parte se añadieron dos ítems que consideramos relevantes para la investigación. En concreto, si las personas mayores tienen menos interés por las relaciones sexuales y si ya no disfrutan con las relaciones sexuales, cuestiones evidentemente necesarias en un estudio relacionado con la percepción de la sexualidad.

Por tanto, el cuestionario final quedó formado por 13 preguntas cerradas (tabla 1). Cada pregunta tiene como posible respuesta 4 sentencias cuyo formato sigue el modelo Likert de cuatro escalas (muy en desacuerdo, en desacuerdo, de acuerdo y muy de acuerdo). A los valores perdidos se les asignó el código 99. Los cambios realizados se validaron a partir de dos procesos distintos: la primera versión del cuestionario fue discutida con 2 expertos en la materia, experimentados en la orientación de investigaciones y amplia práctica educativa en el ámbito de la formación inicial. Se sugirieron reformulaciones relativas al uso del lenguaje adecuado a la realidad educativa, entre ellas el uso de "personas mayores" en vez de "ancianos", tal y como se enuncia en el cuestionario original a este colectivo. Después de la reformulación, se realizó un pre-test con 6 alumnos en formación inicial, no participantes de la muestra, que manifestaron juicios sobre la claridad, la relevancia, la adecuación de las cuestiones, el tiempo necesitado en su realización y el aspecto visual (Bell, 1997; Hill y Hill, 2002). Se tienen en cuenta las sugerencias realizadas por este alumnado con respecto al formato de presentación del cuestionario. El cuestionario final quedó formado por 13 preguntas cerradas. Una vez elaborado y validado el cuestionario por los expertos y la experiencia piloto, para evaluar la confiabilidad tipo consistencia interna, se calculó el coeficiente de alfa de Cronbach. El análisis de fiabilidad de las puntuaciones del cuestionario dio un Coeficiente Alfa de Cronbach de ,792. Como señalan Celina y Campo (2005:577), "el valor mínimo aceptable para el coeficiente alfa de Cronbach es 0,7; por debajo de ese valor la consistencia interna de la escala utilizada es baja". 
Tabla 1: Cuestionario de actitudes hacia la sexualidad en la vejez (CASV) (3-13) y preguntas insertadas (1-2).

\begin{tabular}{|c|c|c|c|c|c|}
\hline \multicolumn{6}{|c|}{1 = Muy en desacuerdo; 2 = Algo en desacuerdo; 3 = Algo de acuerdo; 4 = Muy de acuerdo } \\
\hline \multicolumn{2}{|r|}{ Ítems } & 1 & 2 & 3 & 4 \\
\hline 1 & Las personas mayores tienen menos interés por las relaciones sexuales. & & & & \\
\hline 2 & Las personas mayores ya no disfrutan con las relaciones sexuales. & & & & \\
\hline 3 & Las muestras de amor entre las personas mayores se ven ridículas. & & & & \\
\hline 4 & Las personas mayores deben reprimir su actividad sexual. & & & & \\
\hline 5 & Las personas mayores tienen derecho al amor y la vida sexual. & & & & \\
\hline 6 & Los hombres mayores son impotentes. & & & & \\
\hline 7 & Todas las mujeres mayores son frígidas. & & & & \\
\hline 8 & Las personas mayores ya no tienen deseos sexuales y menos actividad sexual. & & & & \\
\hline 9 & Los cambios que trae el envejecimiento impiden tener actividad sexual. & & & & \\
\hline 10 & Los tratamientos médicos en la vejez complican la actividad sexual. & & & & \\
\hline 11 & La belleza y la sexualidad son exclusivas de los jóvenes. & & & & \\
\hline 12 & La sexualidad se ejerce solo con fines reproductivos. & & & & \\
\hline 13 & Todos los ancianos que desean relacionarse sexualmente son viejos verdes. & & & & \\
\hline
\end{tabular}

\section{Procedimiento}

La recogida de datos se produjo en el curso académico 2016/2017. Todos los participantes fueron debidamente informados de la naturaleza de la investigación, se garantizó el anonimato y la confidencialidad de los datos recogidos. El cuestionario se aplicó dentro del horario lectivo con el visto bueno del responsable docente del aula, siendo personal responsable de la investigación quién introdujo el instrumento y supervisó su cumplimentación. Para ello se necesitó una hora dentro de cada aula en la que se llevó a cabo la recogida de datos. El primer cuarto de hora se dedicó a la presentación de la investigación e instrucciones de cumplimentación del cuestionario, así como a solventar las dudas que pudieran plantearse. El tiempo restante se dedicó a la realización del mismo por parte del estudiantado seleccionado. La duración media que el estudiantado empleó para la realización del cuestionario osciló entre los 30 y los 45 minutos. Para el posterior análisis de los datos recogidos se recurrió al programa informático Statistical Package for the Social Sciences (versión 24, IBM SPSS).

\section{RESULTADOS}

El análisis de los datos se ha realizado teniendo presente que se pueden agrupar en cuatro categorías en función al nivel de estereotipos que se presenta: bajo, medio, alto o muy alto. Para ello, se ha tenido presente la distribución de las respuestas desde una perspectiva dicotómica, que corresponden a una posición afín al estereotipo planteado y una posición opuesta a éste. Las posibilidades han sido:

Nivel bajo: cuando el porcentaje opuesto al estereotipo se sitúa entre el $85 \%$ y el $100 \%$. Nivel medio: cuando el porcentaje opuesto al estereotipo se encuentra entre el $70 \%$ y el $84 \%$. Nivel alto: cuando el porcentaje opuesto al estereotipo se sitúa entre el 55\% y el $69 \%$. Muy alto: cuando el porcentaje opuesto al estereotipo se sitúa entre el $40 \%$ y el $54 \%$.

No se han detectado diferencias significativas entre las respuestas proporcionadas por hombres y mujeres. Dentro de los niveles muy altos de estereotipos solamente se ha situado el ítem 1. Los datos señalan que al cuestionar sobre si las personas mayores tienen menos interés por las relaciones sexuales, los encuestados concentran sus respuestas en los valores centrales. Se entiende que este ítem se encuentra en un nivel muy alto de estereotipo. Un $44 \%$ se encuentran algo de acuerdo, frente a un $34,9 \%$ que se encuentra algo en desacuerdo. Un $13,8 \%$ se considera muy en desacuerdo y un $7,3 \%$ se identifica con el muy de acuerdo. Aunque un $44 \%$ considera que está algo de acuerdo en que las personas mayores tienen menos interés por las relaciones sexuales, las repuestas muy en desacuerdo y algo en desacuerdo, que suman un $48,7 \%$ frente a las respuestas muy de acuerdo y algo de acuerdo unidas, que suman un $51,3 \%$. Podemos afirmar que un $51,3 \%$, más de la mitad de los estudiantes encuestados muestran el estereotipo de que las personas mayores tienen menos interés por las relaciones sexuales (tabla 2).

Tabla 2: Ítems que indican un grado muy alto de estereotipos sobre la sexualidad de personas adultas mayores.

1 = Muy en desacuerdo; 2 = Algo en desacuerdo; 3 = Algo de acuerdo; 4 = Muy de acuerdo

\begin{tabular}{|c|c|c|c|c|c|c|c|c|c|}
\hline \multirow{2}{*}{\multicolumn{2}{|c|}{ Ítems }} & \multicolumn{2}{|c|}{1} & \multicolumn{2}{|c|}{2} & \multicolumn{2}{|c|}{3} & \multicolumn{2}{|c|}{4} \\
\hline & & $F$ & $\%$ & $f$ & $\%$ & $f$ & $\%$ & $f$ & $\%$ \\
\hline P1 & $\begin{array}{l}\text { Las personas mayores tienen menos interés } \\
\text { por las relaciones sexuales. }(\mathrm{N}=109)\end{array}$ & 15 & 13,8 & 38 & 34,9 & 48 & 44,0 & 8 & 7,3 \\
\hline
\end{tabular}


En cuanto a los niveles altos de estereotipos, destaca el ítem 10 que hace referencia a los tratamientos médicos en la vejez como factor que impide una actividad sexual plena a ciertas edades. En este sentido, los porcentajes se reparten entre las respuestas a favor y en contra. Un $48,6 \%$ se manifiesta algo en desacuerdo, frente a un $35,8 \%$ que lo hace como algo de acuerdo. No obstante, si tenemos presente el $13,8 \%$ de muy en desacuerdo y el $1,8 \%$ del muy de acuerdo, comprobamos como el alumnado se manifiesta en desacuerdo con dicha afirmación (muy en desacuerdo-13,8\%, algo en desacuerdo-48,6\%) con un total, de $62,4 \%$. Por otro lado, encontramos un $37,6 \%$ que se manifiesta a favor (algo de acuerdo-35,8\%, muy de acuerdo-1,8\%) (Tabla 3).

Tabla 3: Ítems que indican un grado alto de estereotipos sobre la sexualidad de personas adultas mayores.

\begin{tabular}{|c|c|c|c|c|c|c|c|c|c|}
\hline \multicolumn{10}{|c|}{1 = Muy en desacuerdo; 2 = Algo en desacuerdo; 3 = Algo de acuerdo; 4 = Muy de acuerdo } \\
\hline \multirow{2}{*}{\multicolumn{2}{|c|}{ Ítems }} & \multicolumn{2}{|c|}{1} & \multicolumn{2}{|c|}{2} & \multicolumn{2}{|c|}{3} & \multicolumn{2}{|c|}{4} \\
\hline & & $\mathrm{F}$ & $\%$ & f & $\%$ & f & $\%$ & f & $\%$ \\
\hline P10 & $\begin{array}{l}\text { Los tratamientos médicos en la vejez } \\
\text { complican la actividad sexual. }(\mathrm{N}=109)\end{array}$ & 15 & 13,8 & 53 & 48,6 & 39 & 35,8 & 2 & 1,8 \\
\hline
\end{tabular}

En un nivel medio de estereotipo se encuentran cuatro de los ítems (2, 6, 8 y 9) planteados en el cuestionario a la muestra analizada (tabla 4). Cuando se formula la cuestión sobre si las personas mayores ya no disfrutan con las relaciones sexuales (ítem 2), un $48,1 \%$ se pronuncia como algo en desacuerdo, junto a un $32,4 \%$ que responde que muy en desacuerdo, sumando un total de $80,5 \%$. Por otro lado, un $15,7 \%$ indica que se encuentra algo de acuerdo y un 3,7\% responde que se encuentra muy de acuerdo. Podemos afirmar que un $80,5 \%$ consideran que las personas mayores aún disfrutan con las relaciones sexuales. En cuanto al ítem 6 , para el alumnado encuestado los hombres mayores no son impotentes $(80 \%)$. Ante la afirmación de que los hombres mayores son impotentes, un $40,9 \%$ afirma que se encuentra muy en desacuerdo, un $39,1 \%$ se encuentra algo en desacuerdo, frente a un $15,5 \%$ que afirma estar algo de acuerdo y un $4,5 \%$ que manifiesta estar muy de acuerdo. Por su parte, en el ítem 8 , cuando se plantea que las personas mayores ya no tienen deseos sexuales y menos actividad sexual, un $41,3 \%$ afirma que se encuentra muy en desacuerdo, un $36,7 \%$ se encuentra algo en desacuerdo, un $17,4 \%$ se encuentra algo de acuerdo y un $4,6 \%$ se encuentra muy de acuerdo. Por otro lado, en el ítem 9, al cuestionar sobre si los cambios que trae el envejecimiento impiden tener actividad sexual un $40,9 \%$ indica que encuentra muy en desacuerdo, un $37,3 \%$ se considera algo en desacuerdo, un $20 \%$ algo de acuerdo y un $1,8 \%$ muy de acuerdo.

Tabla 4: Ítems que indican un grado medio de estereotipos sobre la sexualidad de personas adultas mayores.

\begin{tabular}{|c|c|c|c|c|c|c|c|c|c|}
\hline \multicolumn{2}{|c|}{1 = Muy en desacuerdo; 2 = Algo en desacuerdo; 3 = Algo de acuerdo; 4 = Muy de acuerdo } \\
\cline { 2 - 11 } & \multicolumn{1}{|c|}{ Ítems } & $F$ & $\%$ & $f$ & $\%$ & $f$ & $\%$ & $f$ & $\%$ \\
\hline P2 & $\begin{array}{l}\text { Las personas mayores ya no disfrutan con } \\
\text { las relaciones sexuales. (N=108) }\end{array}$ & 35 & 32,4 & 52 & 48,1 & 17 & 15,7 & 4 & 3,7 \\
\hline P6 & $\begin{array}{l}\text { Los hombres mayores son impotentes. } \\
\text { (N=110) }\end{array}$ & 45 & 40,9 & 43 & 39,1 & 17 & 15,5 & 5 & 4,5 \\
\hline P8 & $\begin{array}{l}\text { Las personas mayores ya no tienen deseos } \\
\text { sexuales y menos actividad sexual. (N=109) }\end{array}$ & 45 & 41,3 & 40 & 36,7 & 19 & 17,4 & 5 & 4,6 \\
\hline P9 & $\begin{array}{l}\text { Los cambios que trae el envejecimiento } \\
\text { impiden tener actividad sexual. (N=110) }\end{array}$ & 45 & 40,9 & 41 & 37,3 & 22 & 20,0 & 2 & 1,8 \\
\hline
\end{tabular}

Se ha detectado que en siete de los ítems $(3,4,5,7,11,12$ y 13) el grado de estereotipo es bajo, teniendo presente que más del $90 \%$ de los sujetos encuetados se muestran muy en desacuerdo o algo en desacuerdo con cada estereotipo planteado (tabla 5).

Ante la afirmación sobre si las muestras de amor entre las personas mayores se ven ridículas (ítem 3) un 90\% de los encuestados responden que se encuentran muy en desacuerdo, seguido por un $7,3 \%$ que se consideran algo en desacuerdo, un 1,8\% que se encuentra algo de acuerdo y un $0,9 \%$ que expresa estar muy de acuerdo. En este caso, comprobamos la rotundidad de las respuestas que en un $97,3 \%$ consideran que las muestras de amor entre las personas mayores no se ven ridículas. Para el ítem 4 , un $98,1 \%$ considera que las personas mayores no deben reprimir su actividad sexual. En concreto, un $80,7 \%$ se considera muy en desacuerdo con que deben reprimir su actividad sexual, un 17,4\% se encuentra algo en desacuerdo, un $0,9 \%$ algo de acuerdo y un $0,9 \%$ muy de acuerdo.

Por su parte, en el ítem 5, cuando se afirma que las personas mayores tienen derecho al amor y la vida sexual un $92,7 \%$ se encuentra muy de acuerdo, junto a un $5,5 \%$ que se muestran algo de acuerdo, haciendo un total 
de $98,2 \%$. Por otro lado, un $0,9 \%$ se considera algo en desacuerdo y un $0,9 \%$ responde como muy en desacuerdo. En cuanto al ítem 7, el estudiantado que ha participado considera que las mujeres mayores no son frígidas $(98,2 \%)$. Ante la afirmación de que todas las mujeres mayores son frígidas un $60 \%$ se muestran muy en desacuerdo, un $38,2 \%$ algo en desacuerdo, un 1,8\% algo de acuerdo y no se ha recabado ninguna respuesta muy de acuerdo con dicha afirmación.

Un $82,7 \%$ de los encuestados indican estar muy en desacuerdo con la afirmación de que la belleza y la sexualidad son exclusivas de los jóvenes (ítem 11) y un 14,5\% se encuentran algo en desacuerdo. Por otro lado, un 2,7\% consideran que están algo de acuerdo con esta afirmación. Al afirmar que la sexualidad se ejerce solo con fines reproductivos (Ítem 12) un rotundo 89\% indican estar muy en desacuerdo, junto con un $9,2 \%$ que se encuentra algo en desacuerdo. Por otro lado, encontramos un $0,9 \%$ que indica estar algo de acuerdo con dicha afirmación, junto con un $0,9 \%$ que señala estar muy de acuerdo con la misma.

Ante la cuestión de que todos los ancianos que desean relacionarse sexualmente son viejos verdes (ítem 13), encontramos que un $82,6 \%$ se encuentra muy en desacuerdo con la afirmación, junto con un $14,7 \%$ que se encuentra algo en desacuerdo, llegando a un total, de $97,3 \%$. Por otro lado, un $2,8 \%$ afirma estar algo de acuerdo en que todos los ancianos que desean relacionarse sexualmente son viejos verdes.

Tabla 5: Ítems que indican un grado bajo de estereotipos sobre la sexualidad de personas adultas mayores.

\begin{tabular}{|c|c|c|c|c|c|c|c|c|c|}
\hline \multicolumn{10}{|c|}{1 = Muy en desacuerdo; 2 = Algo en desacuerdo; 3 = Algo de acuerdo; 4 = Muy de acuerdo } \\
\hline \multirow{2}{*}{\multicolumn{2}{|c|}{ Ítems }} & \multicolumn{2}{|c|}{1} & \multicolumn{2}{|c|}{2} & \multicolumn{2}{|c|}{3} & \multicolumn{2}{|c|}{4} \\
\hline & & $f$ & $\%$ & $f$ & $\%$ & $f$ & $\%$ & $F$ & $\%$ \\
\hline P3 & $\begin{array}{l}\text { Las muestras de amor entre las personas } \\
\text { mayores se ven ridículas }(N=110)\end{array}$ & 99 & 90,0 & 8 & 7,3 & 2 & 1,8 & 1 & ,9 \\
\hline P4 & $\begin{array}{l}\text { Las personas mayores deben reprimir su } \\
\text { actividad sexual. }(\mathrm{N}=109)\end{array}$ & 88 & 80,7 & 19 & 17,4 & 1 & 9 & 1 & ,9 \\
\hline P5 & $\begin{array}{l}\text { Las personas mayores tienen derecho al } \\
\text { amor y la vida sexual. }(\mathrm{N}=110)\end{array}$ & 1 & ,9 & 1 & 9 & 6 & 5,5 & 102 & 92,7 \\
\hline P7 & $\begin{array}{l}\text { Todas las mujeres mayores son frígidas. } \\
(\mathrm{N}=110)\end{array}$ & 66 & 60,0 & 42 & 38,2 & 2 & 1,8 & 0 & 0 \\
\hline P11 & $\begin{array}{l}\text { La belleza y la sexualidad son exclusivas de } \\
\text { los jóvenes. }(\mathrm{N}=110)\end{array}$ & 91 & 82,7 & 16 & 14,5 & 3 & 2,7 & 0 & 0 \\
\hline P12 & $\begin{array}{l}\text { La sexualidad se ejerce solo con fines } \\
\text { reproductivos. }(\mathrm{N}=109)\end{array}$ & 97 & 89,0 & 10 & 9,2 & 1 & ,9 & 1 & ,9 \\
\hline P13 & $\begin{array}{l}\text { Todos los ancianos que desean } \\
\text { relacionarse sexualmente son viejos verdes. } \\
(\mathrm{N}=109)\end{array}$ & 90 & 82,6 & 16 & 14,7 & 3 & 2,8 & 0 & 0 \\
\hline
\end{tabular}

\section{DISCUSIÓN}

Se reconoce que en este estudio se presentaron limitaciones que pueden interferir con la generalización de los resultados, la limitación que se consideró de mayor importancia fue que se optó por utilizar un muestreo no probabilístico con la intención de incluir a todos los estudiantes del Grado de Educación Primaria que el día que se presentó y elaboró el cuestionario estuvieran presentes y aceptaran participar en el estudio.

En cuanto a la temática del estudio, se está de acuerdo con Mehrotra, Townsend y Berkman (2009) y Matas et al., (2017) quienes señalan que la población de adultos mayores presenta un desafío para las instituciones de educación superior, especialmente para las que cuentan con programas de Ciencias de la Educación, como pueden ser los Grados de Educación, Pedagogía o Educación Social, que tienen un papel fundamental en la visión social del entorno, y por ende de los colectivos sociales entre los que se encuentra el de los adultos mayores.

Se sabe que los estereotipos son parte de la cultura, sin embargo, cuando son estereotipos negativos por parte de estudiantes de carreras relacionadas con la educación como el Magisterio es preocupante debido a la relevancia social que va a tener su desempeño profesional. De acuerdo con Cerquera, Álvarez y Saavedra 
(2010) y Huici (1999), el comportamiento de los individuos se asocia con los estereotipos y prejuicios y se manifiestan en actitudes negativas e incluso en una serie de conductas que pueden ser discriminatorias hacia un determinado grupo. En este caso, el estudiantado del Grado de Educación Primaria encuestado tiene un perfil medio-bajo de estereotipos negativos hacia la sexualidad en la vejez.

En la revisión de literatura se han encontrado estudios que abordan los estereotipos acerca de la sexualidad en el colectivo de adultos mayores en el mismo colectivo de adultos mayores (Orozco y Rodríguez, 2006; Melguizo-Herrera et al., 2015), en jóvenes (Orozco y Rodríguez, 2006), en estudiantes universitarios de diferentes disciplinas (Arnold-Cathalifaud, Thumala, Urquiza y Ojeda, 2007; Durán-Badillo, Miranda-Posadas, Cruz-Barrera, Martínez-Aguilar, Gutiérrez-Sánchez y Aguilar-Hernández, 2016; Matas et al., 2017) y alumnado y profesorado de etapas educativas pre-universitarias (Cerquera, Álvarez y Saavedra, 2010; Cerquera, Galvis y Cala, 2012).

A nivel general, se observa que la mayoría del alumnado encuestado tiene una percepción positiva respecto al amor y la sexualidad en la vejez, lo cual coincide con los resultados presentados en otros estudios como los Hernández (2008) y Cerquera, Galvis y Cala (2012), los cuales inciden en que los participantes de sus estudios consideran que las personas mayores tienen derecho a ejercer su sexualidad, lo que coincide con los resultados obtenidos en el presente estudio. Estos datos no coinciden con los recabados por Orozco y Rodríguez (2006) en donde los encuestados eran personas adultas mayores que consideraban no tener derecho al amor y la vida sexual. Los mayores porcentajes de estereotipos corresponden al interés por la sexualidad a ciertas edades y la imposibilidad de mantener relaciones debidos a tratamientos médicos. Sin embargo, los datos arrojados en el presente estudio se centran en el derecho al amor y la sexualidad, aunque podemos interpretar que los encuestados perciben que tanto el interés como el deseo decaen en esta etapa de la vida, lo que coincide con otros estudios (Arnold-Cathalifaud et al., 2007; Sahuenza, 2014; Matas et al., 2017). En comparación con otros resultados obtenidos a través del mismo instrumento y a población universitaria, el alumnado de Magisterio en España tiene un nivel de estereotipos bajo con respecto a la sexualidad de los adultos mayores, lo que no se corresponde con el estudio realizado por Durán-Badillo et al. (2016) a estudiantes universitarios de Psicología en Chile, los cuales rebelan un alto porcentaje de estereotipos negativos, lo que es alarmante si se tiene en cuenta que serán los próximos responsables en cuestiones de salud. Por otro lado, coincida con el estudio realizado por Matas et al., 2017, a estudiantes universitarios de magisterio en cuanto al decaimiento del interés y el deseo en esta etapa de la vida. Estos resultados permiten ampliar el conocimiento respecto al tema de estudio y abren la puerta a nuevas inquietudes que pueden ser abordadas en investigaciones posteriores.

\section{CONCLUSIONES}

En respuesta al objetivo planteado para este estudio, centrado en identificar si el alumnado de magisterio posee estereotipos sobre la sexualidad de las personas adultas mayores, los resultados arrojan que existen estereotipos entre estos estudiantes, aunque con una diversidad de intensidad: bajo, medio, alto y muy alto. Predominan las percepciones estereotipadas en un nivel bajo, aunque existen ítems donde el nivel de estereotipo es medio ( 4 ítems), alto ( 1 ítems) y muy alto ( 1 ítems). Estos resultados son comparables con los de Matas et al. (2017), ya que afirma que los resultados obtenidos en el alumnado de magisterio no presentan la intensidad de estereotipos que presentan otros estudios realizados en la misma línea.

Las representaciones que posee la sociedad sobre los grupos que la integran determinan la forma de relacionarse entre cada uno de sus miembros, de este modo, una visión negativa del envejecimiento puede desencadenar una actitud paternalista hacia el adulto mayor frente a una actitud más realista sobre el proceso de envejecimiento que fomentaría una perspectiva afín al enfoque del envejecimiento activo. Identificar los estereotipos e intervenir para afrontarlos forma parte de la prevención (Matas et al., 2017). Esta prevención debe comenzar por los agentes socializadores, en este caso se refiere a los educadores como agentes socializadores y, en concreto en el perfil analizado en este estudio, a los educadores que profesionalmente tienen la opción de ser educadores de personas adultas.

El alumnado encuestado considera que los adultos mayores tienen derecho al amor y al ejercicio de su sexualidad, al igual que en el estudio de Cervera et al. (2012) los adultos mayores aún disfrutan de las relaciones sexuales, no consideran ridículas las muestras de amor, no consideran que deban reprimir su actividad sexual. De igual modo, los encuestados entienden que las personas adultas mayores tienen derecho al amor y la vida sexual, los hombres mayores no son impotentes y las mujeres mayores no son frígidas. Es importante el hecho de que se reconozca el derecho al amor y la vida sexual de este colectivo, así como que tienen deseos sexuales. Por otro lado, es relevante el reconocimiento de que la belleza no es cuestión de edad y que la sexualidad no está ligada a fines reproductivos. Tal y como afirma Matas et al. (2017:75), "Los estudiantes de Educación deberán estar preparados para atender estas necesidades formativas de los adultos mayores" de este modo, el sistema educativo en todas sus etapas, incluida la universitaria, debe contemplar 
la promoción del cambio de actitudes hacia las personas adultas mayores en los diseños curriculares, permitiendo al centro educativo ser parte integrante y activa de la sociedad (Matas et al., 2017; Melero, 2007; Pons, 2011). Debemos tener presente que las personas adultas mayores son agentes activos y participativos de la sociedad y, por ello, deben ser tratados con el menor grado de estereotipos posible en todas sus facetas. Y es que, "la formación de los profesionales se inicia desde las aulas, por tal motivo es necesario enfatizar la identificación de estereotipos negativos hacia la vejez en los estudiantes. Así mismo, se sugiere en futuras investigaciones incursionar en la evaluación de estereotipos hacia la vejez en los profesores de ésta misma área" (Duran-Badillo et al., 2016: 208).

\section{REFERENCIAS}

Aguirre, S., Ornelas, M., Gastélum, G. y Peinado J., Invarianza Factorial de la Escala de Ansiedad ante el Envejecimiento de Lasher y Faulkender en Estudiantes Universitarios, Hombres y Mujeres, doi: 10.4067/S0718-50062017000100004, Formación Universitaria, (en línea), 10 (1), 25-32, (2017)

Allan L., Johnson J. y Emersona S., The role of individual difference variables in ageism, doi: 10.1016/j.paid.2013.10.027, Personality and Individual Differences, (por suscripción), 59, 32-37, (2014)

Amador, L., Malagón, J. L. y Mateos F., Los estereotipos en la vejez en A. J. Colom y C. Orte, Gerontología educativa y social. Pedagogía social y personas mayores, $1^{\underline{a}}$ Ed., Universitat de les Illes Balears (pp. 57-76). Palma de Mallorca, España, (2001)

Amador, L., Moreno-Crespo, P. y Cárdenas, R., Envejecimiento y sociodemografía mundial en J.L. Malagón, V. Pérez y L. Amador (Dir.), Vejez, autonomía o dependencia, pero con calidad de vida, 1a Ed., Dykinson, Madrid, España, (2006)

Arnold-Cathalifua, M., Thumala, D., Urquiza, A. y Ojeda, A., La vejez desde la mirada de los jóvenes chilenos: estudio exploratorio, Última Década, 27, 75-91, (2007)

Bárcena, C., Iglesias, J.A., Galán, Ma․I y Abella, V., Dependencia y edadismo: implicaciones para el cuidado, Revista Enfermería CyL, 1 (1), 46-52, (2009)

Bell, J., Como realizar un projecto de investigação, 1르. Ed., Lisboa, Gradiva, (1997)

Bergman Y. y Bodner E., Ageist attitudes block young adults' ability for compassion toward incapacitated older adults, doi: https://doi.org/10.1017/S1041610215000198, International Psychogeriatrics, (en línea), 27(9), 1541-1550 (2015)

Canales, M., Metodologías de investigación social, 1르 Ed., LOM Ediciones, Santiago, Chile, (2006)

Celina, H. y Campo, A., Aproximación al uso del coeficiente alfa de Cronbach, Revista Colombiana de Psiquiatría, (en línea), XXXIV (4), 572-580, (2005)

Cerquera, A. M., Alvarez, J. L., y Saavedra, A.C., Identificación de estereotipos y prejuicios hacia la vejez presentes en una comunidad educativa de Floridablanca, Psychologia: Avances de la Disciplina, 4 (1), 73-87, (2010)

Cerquera, A.M. Galvis, M. J. y Cala M.L., Amor, sexualidad e inicio de nuevas relaciones en la vejez: percepción de tres grupos etarios, doi: http: //dx.doi.org/10.21500/19002386.1185, Psychologia: avances de la Disciplina, (en línea), 6(2), 7381, (2012)

De Lemus, S., Estereotipos y prejuicio de género: automatismo y modulación contextual. Tesis Doctoral, Granada, España, (2007)

Díaz, A., Fernández, M., Gázquez, J., González, L., Pérez-Fuentes, M. y Ruíz, I., Oldage stereotypes related to gerontology education: An intergenerational study. European Journal of Education and Psychology, 2 (3), 263-273, (2009)

Durán-Badillo, T., Miranda-Posadas, C., Cruz-Barrera, L., Martínez-Aguilar, Mª . L., Gutiérrez-Sánchez, G. y AguilarHernández, R. M., Estereotipos negativos sobre la vejez en estudiantes universitarios de enfermería, Rev. Enferm. Inst. Mex. Seguro Soc., 24 (3), 205-209, (2016)

Escarbajal, A. y Martínez S. M., Jubilación, educación y calidad de vida, Pedagogía Social: revista interuniversitaria, ISSN-e: 1989-9742 (en línea), 20, 245-272, (2012), https://goo.gl/5k4kUG. Acceso: 7 de Julio (2017)

Escarbajal, A., Martínez, S. M. y Salmerón J.A., La percepción de la calidad de vida en las mujeres mayores y su envejecimiento activo a través de actividades socioeducativas en los centros sociales, doi: 10.6018/rie.33.2.213211, Revista de Investigación Educativa, (en línea), 33(2), 471-488, (2015)

Fernández-Ballesteros, R. (Dir.) Gerontología Social, 1aㅡ. Ed., Pirámide, Madrid, España, (2000)

Freixas, A. y Luque, B. El secreto mejor guardado: la sexualidad de las mujeres mayores, Política y sociedad, 46 (1 y 2 ), 191-203, (2009)

García-Martínez, J. A. y Benito J. (Coords.) Educación para la Salud y Personas Mayores. 1ª Ed., Ayuntamiento de San Pedro del Pinatar, Murcia, España, (2003)

García-Martínez, J.A. Benito, J., Martínez, R., Lidón, B., Sánchez, A. y Pedrero, E., Las personas mayores desde la perspectiva educativa para la salud, 1aㅡ., Universidad de Murcia, Murcia, España, (2002)

Hernández, R., Fernández, C. y Baptista P., Metodología de la investigación, 4ª Ed., McGraw Hill, México, (2006) 
Hernández, Z., Algunos aspectos a considerar sobre la sexualidad en el adulto mayor, El Ágora USB, 8, 375-387, (2008) Hill, M. M., y Hill, A., Investigaçao por questionário, $1^{a}$ Ed., Lisboa, Ediçoes Sílabo, (2002)

Huici, C., Estereotipos, en Morales, J.F. (Coord.), Psicología Social, 2ª Ed., Madrid, McGraw-Hill, 87-98, (1999)

Leiva, V., Arguedas, C., Hidalgo, M. y Navarro Y., Conocimiento de las personas adultas sobre el climaterio, andropausia y la sexualidad, Rev. Ciencias Sociales, (II), 163-173, (2013)

Levy, B.R. y Myers L.M., Preventive health behaviors influenced by self-perceptions of aging, doi: 10.1016/j.ypmed.2004.02.029, (por suscripción), Preventive Medicine, 39 (3), 625-629, (2004)

López-Sánchez, F. y Olozabal J.C., Sexualidad en la vejez, 3aㅡ Ed., Pirámide, Madrid, España, (2005)

López-Sánchez, F., Sexualidad y afectos en la vejez, 1aㅡ. Ed., Pirámide, Madrid, España, (2012)

Lucas-Matheu, M., Sexualidad madura, 1aㅡ edición, Síntesis, Madrid, España, (2013)

Matas, A., Leiva, J. y Franco, P.D., Análisis del uso de las Nuevas Tecnologías en población española de 45-54 años: previsión de necesidades formativas para un envejecimiento activo, Pixel-Bit, 48, 225-240, (2016)

Matas, A., Leiva, J.J., Franco, P.D. e Isequilla, E., Representación social de los estudiantes de magisterio sobre los mayores: un estudio piloto, Rev. Electrónica de Investigación y Docencia (REID), 17, 61-78, (2017)

Mehrotra, C., Townsend, A., y Berkman, B., Enhancing research capacity in gerontological social work, Educational Gerontology, 35(2), 146-163, (2009)

Melero, L., Modificaciones de los estereotipos sobre los mayores, Comunicación e persoas maiores: Actas do Foro Internacional, 29-46, (2007)

Melguizo-Herrera, E., Álvarez-Romero, Y., Cabarcas-Mendoza, M.V., Calvo-Rodríguez, R.S., Flórez-Almanza, J. MoadieContreras, O.P y Campo-Arias, A., Validez y confiabilidad del cuestionario de actitudes hacia la sexualidad en la vejez en adultos mayores en Cartagena, Colombia, Revista Colombiana de Psiquiatría, 44 (2), 87-92, (2015)

Moragas, R. y Linz J.J., Gerontología Social: envejecimiento y calidad de vida, 1르 Ed., Herder, Barcelona, España, (1991) Morales, J.F y Moya, M.C., Tratado de psicología social. Vol. I: procesos básicos, Síntesis, Madrid, España, (1996)

Moreno-Crespo, P. y Pérez-Pérez, I., Estereotipos sobre la jubilación en pretitulados universitarios. (En línea: http://www.ub.edu/ice/reire.htm. Acceso: 4 de julio de 2017), REIRE, Revista d'Innovació i Recerca en Educació, (2014)

Moreno-Crespo, P., Educación a lo largo de la vida: aula de mayores. En línea: http://dx.doi.org/10.12795/revistafuentes.2015.i17.05. Acceso: 4 de julio de 2017, Revista Fuentes, (2015)

Moreno-Crespo, P., Mayores y aprendizaje: envejecimiento activo, IX Congreso Nacional de Organizaciones de Mayores. "Arte de Envejecer", CEOMA, Madrid, España, (2010)

Moreno-Crespo, P., Mayores y Formación. Aprendizaje y Calidad de Vida. Tesis Doctoral (no publicada), Universidad Pablo de Olavide, Sevilla, España, (2011).

Orozco, I. y Rodríguez, D.D., Prejuicios y actitudes hacia la sexualidad en la vejez, Psicología y Ciencia Social. Red de Revistas Científicas de América Latina y el Caribe, España y Portugal. Universidad Nacional Autónoma de México, ISSN: 1405-5082 (en línea), 8(1), 3-10, 2006, https://goo.gl/DTTm1r. Acceso: 7 de Julio, (2017)

Pedrero, E., La sexualidad en las personas mayores en A. García-Martínez y J. Benito, Educación para la Salud y Personas Mayores.1 ${ }^{a}$ Ed., Ayto. de San Pedro del Pinatar, pp. 189-205, Murcia, España, (2003)

Pedrero, E., Los mayores: una nueva edad adulta en el siglo XXI. Trabajo monográfico para el Curso Virtual: Educación para el Envejecimiento. En línea: https://goo.gl/GnnNfb. Acceso: 4 de julio de 2017. Revista Tiempo. El Portal de la Psicogerontología, (2002)

Pons, R.M., Editorial Comment: Education and Prejudice. Anales de Psicología, 3(27), 582-586, (2011)

Sahuenza, J., Imágenes sobre la vejez en jóvenes estudiantes de carreras sin formación gerontológica, Neurama, Revista electrónica de Psicogerontología, 1(1), 6-14, (2014)

Sapetti, A., La sexualidad en el adulto mayor, Psicodebate. Psicología, Cultura y Sociedad, 13, 61-78, (2013)

Triadó, C. y Villar F., Psicología de la vejez, 1a Ed., Alianza, Madrid, España, (2006)

Van Dussen, D y Weaver, R., Undergraduate students' perceptions and behaviors related to the aged and to aging processes, Educational Gerontology, 35(4), 342-357, (2009)

Vermulen, A., Kaufman, J.M., Ageing of the Hypothalamo-Pituitary-Testucular Axis in Men, Horm Res, 43, 25-28, (1995) 